\title{
THE SIGNLESS LAPLACIAN SPECTRAL RADIUS OF BICYCLIC GRAPHS WITH A GIVEN GIRTH*
}

\author{
ZHONGXUN ZHU ${ }^{\dagger}$
}

\begin{abstract}
Let $B(n, g)$ be the class of bicyclic graphs on $n$ vertices with girth $g$. In this paper, the graphs in $B(n, g)$ with the largest signless Laplacian spectral radius are characterized.
\end{abstract}

Key words. Bicyclic graph, Signless Laplacian matrix, Signless Laplacian spectral radius.

AMS subject classifications. 05C69, 05C05.

1. Introduction. All graphs considered in this paper are finite, undirected and simple. Graph theoretical terms used but not defined can be found in Bollobás [1].

Let $G=(V, E)$ be a connected graph with vertex set $V(G)=\left\{v_{1}, v_{2}, \ldots, v_{n}\right\}$ and edge set $E(G)=\left\{e_{1}, e_{2}, \ldots, e_{m}\right\}$. If $m=n-1+c$, then $G$ is called a $c$-cyclic graph. If $c=0,1$ and 2 , then $G$ is a tree, unicyclic graph, and bicyclic graph, respectively. We denote by $P_{n}, C_{n}$ and $S_{n}$ the path, the cycle and the star on $n$ vertices, respectively. Set $N\left(v_{i}\right)=\left\{u \mid u v_{i} \in E(G)\right\}$ and $N\left[v_{i}\right]=N\left(v_{i}\right) \cup\left\{v_{i}\right\}$. Denote by $d_{G}\left(v_{i}\right)\left(=\left|N\left(v_{i}\right)\right|\right)$, or briefly by $d_{v_{i}}$, the degree of vertex $v_{i}$ of $G$.

The signless Laplacian of a graph $G$ is the matrix $Q(G)=D(G)+A(G)$, where $A(G)$ and $D(G)$ denote respectively the $(0,1)$ adjacency matrix and the diagonal matrix of vertex degrees of $G$. If $R$ is the vertex-edge incidence matrix of $G$, then $Q(G)=R R^{T}$. The matrix $L(G)=D(G)-A(G)$ is known as the Laplacian matrix of $G$. Recently, the matrix $Q(G)$ has attracted the attention of many researchers. Some have expressed the view that, in comparison to the spectra of other commonly used graph matrices (such as the Laplacian and the adjacency matrix), the signless Laplacian seems to be the most convenient for use in studying graph properties [2].

One natural line of research to pursue find upper bounds on the spectral radius of the signless Laplacian and characterize the graphs attaining these bounds. Now many papers on the signless Laplacian spectrum have been published. In [3, 4], X.D. Zhang studied the signless Laplacian spectral radius of trees and unicyclic graphs with given

${ }^{*}$ Received by the editors on September 27, 2010. Accepted for publication on March 18, 2011. Handling Editor: Bryan Shader.

${ }^{\dagger}$ College of Mathematics and Statistics, South Central University for Nationalities, Wuhan 430074, P.R. China (zzxun73@mail.scuec.edu.cn). Supported by the Special Fund for Basic Scientific Research of Central Colleges, South-Central University for Nationalities (No CZY10010). 
degree sequences. In [5], Fan et. al. discussed the signless Laplacian spectral radius of bicyclic graph with fixed order. In [6], the author characterized the extremal graph of bicyclic graphs with $k$ pendant vertices with maximal signless Laplacian spectral radius. For further details, we refer the reader to the recent paper by D. Cvetković [7], which is a wonderful survey on the signless Laplacian, and the cited references therein.

For a connected graph $G$, it is well known that $Q(G)$ is a positive semidefinite matrix, that is, all its eigenvalues are nonnegative. Furthermore, by the PerronFrobeniuns Theorem, the largest eigenvalue $\mu(G)$ of $Q(G)$ is simple and there is a unique positive unit eigenvector $X=\left(X\left(v_{1}\right), \ldots, X\left(v_{n}\right)\right)^{T}$. We shall refer to such an eigenvector as the Perron vector of $Q(G)$.

Let $B(n, g)$ be the class of bicyclic graphs on $n$ vertices with girth $g$. In this paper, we study the signless Laplacian spectral radius and determine the graph with the largest signless Laplacian spectral radius in $B(n, g)$.

2. Preliminaries. In this section, we list some known results which will be used in this paper.

Let $G$ be a connected graph, and $u v \in E(G)$. The graph $G_{u, v}$ is obtained from $G$ by subdividing the edge $u v$, i.e., adding a new vertex $w$ and edges $w u, w v$ in $G-u v$. Hoffman and Smith [8] define an internal path of $G$ as a walk $u_{0} u_{1} \cdots u_{s}(s \geq 1)$ such that the vertices $u_{0}, u_{1}, \ldots, u_{s-1}$ are distinct, $d\left(u_{0}\right)>2, d\left(u_{s}\right)>2$, and $d\left(u_{i}\right)=2$, whenever $0<i<s$. An internal path is closed if $u_{0}=u_{s}$.

LEMMA 2.1. [9, 10] Let $G$ be a connected graph and uv be an edge on an internal path of $G$. Then $\mu\left(G_{u, v}\right)<\mu(G)$.

Lemma 2.2. [11] Let $G$ be a connected graph. Suppose $u_{1}$ and $u_{2}$ are vertices each of degree at least three and $u_{1} u_{2}$ is an edge of $G$. Let $G^{\prime}$ be the connected graph obtained from $G$ by contracting $u_{1} u_{2}$ (i.e., deleting the edge and identifying $u_{1}$ and $\left.u_{2}\right)$. Then $\mu(G)<\mu\left(G^{\prime}\right)$.

Lemma 2.3. [11] Let $G$ be a connected graph and $P$ be a pendant path in $G$. Suppose $e$ is an edge in $P$ and $G^{\prime}$ is the graph obtained from $G$ by subdividing $e$. Then $\mu(G)<\mu\left(G^{\prime}\right)$.

LEMmA 2.4. [11] Let $G$ be a connected graph of order $n$ and $S, T$ be nonempty vertex subsets. Suppose $S=\left\{u_{1}, u_{2}, \ldots, u_{s}\right\}$ and the neighbors of $u_{i}$ in $T$ are $u_{i 1}, u_{i 2}, \ldots$, $u_{i l_{i}}\left(l_{i} \geq 1, i=1,2, \ldots, s\right)$. Let $X=\left(X\left(v_{1}\right), X\left(v_{2}\right), \ldots, X\left(v_{n}\right)\right)^{T}$ be the Perron vector of $Q(G)$, where $X\left(v_{k}\right)$ corresponds to the vertex $v_{k}(1 \leq k \leq n)$. Suppose $X\left(u_{1}\right)=\max \left\{X\left(u_{i}\right): i=1,2, \ldots, s\right\}$. Let $H$ be the graph obtained from $G$ by deleting edges $u_{i} u_{i j}$ and adding the edges $u_{1} u_{i j}\left(i=2,3, \ldots, s, j=1,2, \ldots, l_{i}\right)$. Then 
$\mu(G)<\mu(H)$.

LEMMA 2.5. [12] Let $G$ be a graph on $n$ vertices with at least one edge and the maximum degree of $G$ be $\triangle$. Then $\mu(G) \geq \triangle+1$. The equality holds if and only if $G$ is a star.

Lemma 2.6. [6] For a connected graph $G, \mu(G) \leq \max \left\{d_{u}+m_{u}: u \in V(G)\right\}$, where $m_{u}$ satisfies $d_{u} m_{u}=\sum_{u v \in E(G)} d_{v}$. The equality holds if and only if $G$ is regular or semiregular bipartite.

Lemma 2.7. [13] For a connected graph $G$,

$$
\mu(G) \leq \max \left\{\frac{d_{v_{i}}\left(d_{v_{i}}+m_{v_{i}}\right)+d_{v_{j}}\left(d_{v_{j}}+m_{v_{j}}\right)}{d_{v_{i}}+d_{v_{j}}} \mid v_{i} v_{j} \in E(G)\right\}
$$

where $m_{v_{i}}$ satisfies $d_{v_{i}} m_{v_{i}}=\sum_{v_{i} v_{j} \in E(G)} d_{v_{j}}$.

LEMma 2.8. [5] Let $G$ be a graph with maximal signless Laplacian spectral radius among connected bicyclic graph, then $G$ is the graph obtained from $S_{n}$ by adding two adjacent edges.

3. Main results. In this section, we first consider how the signless Laplacian spectral radius behaves when the graph is perturbed. Then we characterize the graph with the largest signless Laplacian spectral radius in $B(n, g)$.

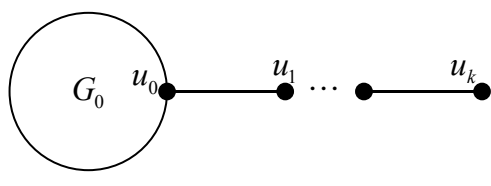

$G_{1}$

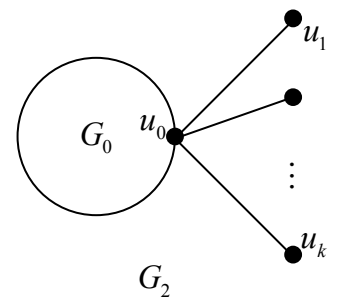

$G_{2}$

Fig. 3.1. The graphs $G_{1}$ and $G_{2}$.

Lemma 3.1. Let $G_{1}, G_{2}$ be graphs of the form in Figure 3.1. If $X$ is the Perron vector of $Q\left(G_{1}\right)$, and $X\left(u_{0}\right) \geq X\left(u_{i}\right), i=1,2, \ldots, k(k \geq 2)$, then $\mu\left(G_{2}\right)>\mu\left(G_{1}\right)$.

Proof. Let $R$ be the vertex-edge incidence matrix of $G_{1}$. Since $X$ is the Perron vector of $Q\left(G_{1}\right)$, then

$$
\begin{aligned}
\mu\left(G_{1}\right) & =X^{T} Q\left(G_{1}\right) X=X^{T} R R^{T} X=\left(R^{T} X\right)^{T} R^{T} X \\
& =\sum_{v_{i} v_{j} \in E\left(G_{1}\right), 1 \leq i<j \leq n}\left(X\left(v_{i}\right)+X\left(v_{j}\right)\right)^{2} .
\end{aligned}
$$


Obviously, $E\left(G_{2}\right)-\left\{u_{0} u_{2}, u_{0} u_{3}, \ldots, u_{0} u_{k}\right\}=E\left(G_{1}\right)-\left\{u_{1} u_{2}, u_{2} u_{3}, \ldots, u_{k-1} u_{k}\right\}$. Then

$$
\begin{aligned}
& X^{T} Q\left(G_{2}\right) X-X^{T} Q\left(G_{1}\right) X \\
= & \sum_{v_{i} v_{j} \in E\left(G_{2}\right), 1 \leq i<j \leq n}\left(X\left(v_{i}\right)+X\left(v_{j}\right)\right)^{2}-\sum_{v_{i} v_{j} \in E\left(G_{1}\right), 1 \leq i<j \leq n}\left(X\left(v_{i}\right)+X\left(v_{j}\right)\right)^{2} \\
= & \left(X\left(u_{0}\right)+X\left(u_{2}\right)\right)^{2}+\cdots+\left(X\left(u_{0}\right)+X\left(u_{k}\right)\right)^{2}-\left[\left(X\left(u_{1}\right)+X\left(u_{2}\right)\right)^{2}+\cdots\right. \\
& \left.+\left(X\left(u_{k-1}\right)+X\left(u_{k}\right)\right)^{2}\right] \\
= & {\left[\left(X\left(u_{0}\right)+X\left(u_{2}\right)\right)^{2}-\left(X\left(u_{1}\right)+X\left(u_{2}\right)\right)^{2}\right]+\cdots+\left[\left(X\left(u_{0}\right)+X\left(u_{k}\right)\right)^{2}\right.} \\
& \left.-\left(X\left(u_{k-1}\right)+X\left(u_{k}\right)\right)^{2}\right] \\
\geq & 0
\end{aligned}
$$

since $X\left(u_{0}\right) \geq X\left(u_{i}\right)>0, i=1,2, \ldots, k$. So

$$
\mu\left(G_{2}\right)=\max _{Y \in R^{n},\|Y\|=1} Y^{T} Q\left(G_{2}\right) Y \geq X^{T} Q\left(G_{2}\right) X \geq X^{T} Q\left(G_{1}\right) X=\mu\left(G_{1}\right) .
$$

If $\mu\left(G_{2}\right)=\mu\left(G_{1}\right)$, then $Q\left(G_{1}\right) X=\mu\left(G_{1}\right) X$ and $Q\left(G_{2}\right) X=\mu\left(G_{2}\right) X$. Thus,

$$
\begin{gathered}
\mu\left(G_{1}\right) X\left(u_{0}\right)=\left(d_{G_{0}}\left(u_{0}\right)+1\right) X\left(u_{0}\right)+\sum_{u u_{0} \in E\left(G_{0}\right)} X(u)+X\left(u_{1}\right), \\
\mu\left(G_{2}\right) X\left(u_{0}\right)=\left(d_{G_{0}}\left(u_{0}\right)+k\right) X\left(u_{0}\right)+\sum_{u u_{0} \in E\left(G_{0}\right)} X(u)+\sum_{i=1}^{k} X\left(u_{i}\right),
\end{gathered}
$$

so we have $\mu\left(G_{2}\right)>\mu\left(G_{1}\right)$, a contradiction. Hence, $\mu\left(G_{2}\right)>\mu\left(G_{1}\right)$.

If $H_{1}, H_{2}$ are graphs with $V\left(H_{1}\right) \cap V\left(H_{2}\right)=v$, then $G=H_{1} v H_{2}$ is defined as a new graph with $V(G)=V\left(H_{1}\right) \cup V\left(H_{2}\right)$ and $E(G)=E\left(H_{1}\right) \cup E\left(H_{2}\right)$. We always assume that in graph $G v S_{l}, v$ is identified with the center of the star $S_{l}$ in $G v S_{l}$.

LEMMA 3.2. Let $H$ be a graph and $T_{l} \neq S_{l}$ be a tree of order l with $V(H) \cap V\left(T_{l}\right)=$ $v$. Then $\mu\left(H v S_{l}\right)>\mu\left(H v T_{l}\right)$.

Proof. Since $T_{l} \neq S_{l}$, it follows that $l \geq 3$.

Case 1. $T_{l}=P_{l}$.

Let $P_{l}=v v_{1} \cdots v_{l-1}, N_{H v P_{l}}(v) \backslash\left\{v_{1}\right\}=\left\{u_{1}, \ldots, u_{s}\right\}$ and $X$ be the Perron vector of $Q\left(H v P_{l}\right)$. Set $X\left(v_{r}\right)=\max \left\{X(w): w \in\left\{v, v_{1}, \ldots, v_{l-1}\right\}\right\}$. If $X\left(v_{r}\right)=X(v)$, then, by Lemma 3.1, we have $\mu\left(H v S_{l}\right)>\mu\left(H v T_{l}\right)$. If $X\left(v_{r}\right) \neq X(v)$, deleting the edges $v u_{i}$ $(i=1, \ldots, s)$, and adding the edges $u_{i} v_{r}, i=1, \ldots, s$, denote the resulted graph by $G_{0}$, by Lemma $2.4, \mu\left(G_{0}\right)>\mu\left(H v T_{l}\right)$. Let $G_{1}$ be the graph obtained $H$ by attaching two pendant paths $P^{1}=v_{r} v_{r+1} \cdots v_{l-1}$ and $P^{2}=v_{r} v_{r-1} \cdots v$ at $v_{r}$, by Lemma 3.1, we have $\mu\left(H v S_{l}\right)>\mu\left(G_{1}\right)$. Obviously, $G_{0} \cong G_{1}$, then $\mu\left(H v S_{l}\right)>\mu\left(H v T_{l}\right)$. 
Case 2. $T_{l} \neq P_{l}$.

First, contracting all internal paths in $T_{l}$, and path $P=v v_{1} \cdots v_{k}$, where $d_{H v T_{l}}(v)$ $\geq 3, d_{T_{l}}\left(v_{i}\right)=2\left(i=1, \ldots, v_{k-1}\right)$ and $d_{T_{l}}\left(v_{k}\right) \geq 3$ (if any), denote the resulted graph by $H v T^{1}$, by Lemma 2.1, $\mu\left(H v T^{1}\right)>\mu\left(H v T_{l}\right)$. Then contracting all edges with degree of each endvertex at least three in $T^{1}$ and edge $v v_{k}$ with $d_{H v T^{1}}(v) \geq 3$ and $d_{T^{1}}\left(v_{k}\right) \geq 3$ (if any), by Lemma 2.2 , the signless Laplacian spectral radius increases. Further, subdividing a pendant path several times if necessary to keep the order of graph unchanged, denote the resulted graph by $G^{\prime}=H v T_{l}^{2}$, by Lemma 2.3 , the signless Laplacian spectral radius also increases. Obviously, $G^{\prime}$ is the graph obtained from $H$ by attaching some pendant paths at $v$. Similar to the proof of Case 1, we have $\mu\left(H v S_{l}\right)>\mu\left(H v T_{l}\right)$.

Let $G$ be a bicyclic graph. The base of $G$, denoted by $B(G)$, is the minimal bicyclic subgraph of $G$. Obviously, $B(G)$ is the unique bicyclic subgraph of $G$ containing no pendant vertex, and $G$ can be obtained from $B(G)$ by planting trees to some vertices of $B(G)$.

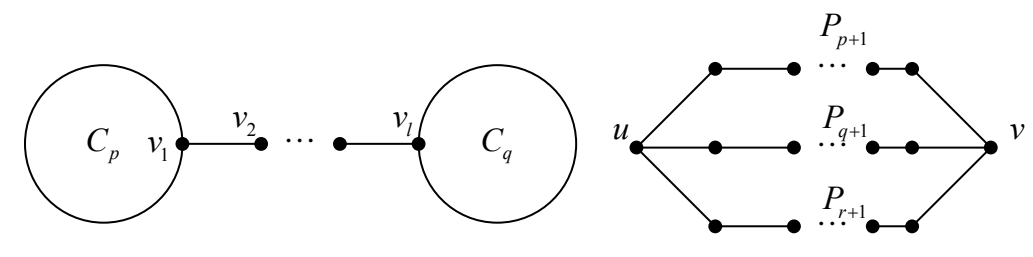

FIG. 3.2. The bases of $B(n, g)$.

It is well known that bicyclic graphs have the following two types of bases (as shown in Figure 3.2):

Let $\widehat{B}(p, l, q)$ be the graph obtained by joining a new path $v_{1} v_{2} \cdots v_{l}$ between two cycles $C_{p}$ and $C_{q}$, where $v_{1} \in V\left(C_{p}\right)$ and $v_{l} \in V\left(C_{q}\right)$. Let also $P(p, q, r)$ be the bicyclic graph consisting of three pairwise internal disjoint paths $P_{p+1}, P_{q+1}, P_{r+1}$ with common endpoints $u, v$.

Now we can define the following two classes of bicyclic graphs on $n$ vertices with a given girth $g$ :

$$
\begin{aligned}
& B_{1}(n, g)=\{G \in B(n, g) \mid B(G)=\widehat{B}(p, l, g) \text { for some } p \geq g \geq 3 \text { and } l \geq 1\} \\
B_{2}(n, g)= & \{G \in B(n, g) \mid B(G)=P(p, q, r) \text { for some } 1 \leq p \leq q \leq r \text { and } \\
& p+q=g \geq 3\} .
\end{aligned}
$$

Then $B(n, g)=B_{1}(n, g) \cup B_{2}(n, g)$. 
LEMMA 3.3. Let $G^{*}$ have the maximal signless Laplacian spectral radius among all graphs in $B(n, g)$. Then $G^{*}$ is obtained from $B\left(G^{*}\right)$ by attaching some pendant edges (if any) to a unique vertex $v^{*}$.

Proof. Note that $G^{*}$ can be obtained from $B\left(G^{*}\right)$ by attaching trees to some vertices of $B\left(G^{*}\right)$. By Lemma 3.2, these trees must be stars, since $G^{*}$ is the extremal graph. Let $\left\{v_{1}, \ldots, v_{t}\right\}$ be the set of centers of these stars and $X$ be the Perron vector of $Q\left(G^{*}\right)$. Set $V_{i}=\left\{v_{i 1}, v_{i 2}, \ldots, v_{i r_{i}}\right\}$ be the set of pendent vertices which are adjacent to $v_{i}, i=1,2, \ldots, t$. If $t \geq 2$, without loss of generality, let $X\left(v_{1}\right)=\max \left\{X\left(v_{i}\right) \mid 1 \leq\right.$ $i \leq t\}$, then by Lemma 2.4 ,

$$
\begin{aligned}
\mu\left(G^{*}\right)< & \mu\left(G^{*}-\left\{v_{2} v_{21}, \ldots, v_{2} v_{2 r_{2}}, \ldots, v_{t} v_{t 1}, \ldots, v_{t} v_{t r_{t}}\right\}\right. \\
& +\left\{v_{1} v_{21}, \ldots, v_{1} v_{2 r_{2}}, \ldots, v_{1} v_{t 1}, \ldots, v_{1} v_{t r_{t}}\right\}
\end{aligned}
$$

a contradiction. Thus, $t=1$. This completes the proof.

TheOREM 3.4. Let $G^{*}$ have the maximal signless Laplacian spectral radius among all graphs in $B_{1}(n, g)$. Then $G^{*} \cong \widehat{B}(g, 1, g) v_{1} S_{n-2 g+2}$ and $\mu\left(G^{*}\right)<n-2 g+6$ $+\frac{4}{n-2 g+5}$.

Proof. Since $G^{*} \in B_{1}(n, g), B\left(G^{*}\right) \cong \widehat{B}(p, l, g)$ for some $l \geq 1, p \geq g$.

Suppose $l \geq 2$ and $P_{l}=v_{1} v_{2} \cdots v_{l}$, where $v_{1} \in V\left(C_{p}\right), v_{l} \in V\left(C_{g}\right)$. Let $X$ be the Perron vector of $Q\left(G^{*}\right)$. Without loss of generality, let $X\left(v_{1}\right) \geq X\left(v_{l}\right)$. By Lemma 2.4 , we have

$$
\mu\left(G^{*}\right)<\mu\left(G^{*}-\sum_{v \in V\left(C_{g}\right) \cap N\left(v_{l}\right)} v v_{l}+\sum_{v \in V\left(C_{g}\right) \cap N\left(v_{l}\right)} v v_{1}\right),
$$

a contradiction. Thus $l=1$, that is, $V\left(C_{g}\right) \cap V\left(C_{p}\right)=\left\{v_{1}\right\}$.

By Lemma $3.3, G^{*}$ is obtained from $B\left(G^{*}\right)$ by attaching some pendant edges (if any) to a unique vertex $v^{*}$. If $v^{*} \neq v_{1}$, comparing $X\left(v^{*}\right)$ and $X\left(v_{1}\right)$, we can similar find a graph in $B_{1}(n, g)$ which has larger signless Laplacian spectral radius than $G^{*}$. Hence, $v^{*}=v_{1}$.

Let $k=n-g-p+1$. If $p \geq g+1$, then $n \geq 2 g$ and $k \leq n-2 g$. By Lemma 2.6, we have

$$
\mu\left(G^{*}\right)<\max \left\{d_{x}+m_{x} \mid x \in V\left(G^{*}\right)\right\}=k+4+\frac{k+8}{k+4}=k+5+\frac{4}{k+4} .
$$

Let $f(k)=k+5+\frac{4}{k+4}$, then $f(k)$ is increasing with nonnegative number $k$. Thus,

$$
\mu\left(G^{*}\right)<n-2 g+5+\frac{4}{n-2 g+4} \leq n-2 g+6 .
$$


However, by Lemma $2.5, \mu\left(\widehat{B}(g, 1, g) v_{1} S_{n-2 g+2}\right) \geq \triangle+1=n-2 g+6>\mu\left(G^{*}\right)$, a contradiction. So $p=g$.

Hence, $G^{*} \cong \widehat{B}(g, 1, g) v_{1} S_{n-2 g+2}$ and

$$
\begin{aligned}
\mu\left(\widehat{B}(g, 1, g) v_{1} S_{n-2 g+2}\right) & <\max \left\{d_{x}+m_{x} \mid x \in V\left(\widehat{B}(g, 1, g) v_{1} S_{n-2 g+2}\right)\right\} \\
& =n-2 g+6+\frac{4}{n-2 g+5} \cdot
\end{aligned}
$$

LEMMA 3.5. Let $G^{*}$ have the maximal signless Laplacian spectral radius among all graphs in $B_{2}(n, g)$. Then $B\left(G^{*}\right) \cong P\left(\left\lfloor\frac{g}{2}\right\rfloor,\left\lceil\frac{g}{2}\right\rceil,\left\lceil\frac{g}{2}\right\rceil\right)$.

Proof. Since $G^{*} \in B_{2}(n, g), B\left(G^{*}\right) \cong P(p, q, r)$, where

$$
1 \leq p \leq q \leq r, \quad p+q=g
$$

Obviously, $n \geq(p+q+r)-1$ and $p+q+r \geq g+\left\lceil\frac{g}{2}\right\rceil$, then $n \geq g+\left\lceil\frac{g}{2}\right\rceil-1$.

Case 1. $n=g+\left\lceil\frac{g}{2}\right\rceil-1$. Then $G^{*}$ cannot contain pendant edges and $p+q+r=$ $g+\left\lceil\frac{g}{2}\right\rceil$. By (3.1), we have $r=\left\lceil\frac{g}{2}\right\rceil$ and $p \leq q \leq\left\lceil\frac{g}{2}\right\rceil$. If $q \leq\left\lceil\frac{g}{2}\right\rceil-1$, then $p \geq\left\lfloor\frac{g}{2}\right\rfloor+1$. Obviously, if $g$ is even, $p \geq \frac{g}{2}+1$ and $q \leq \frac{g}{2}-1$, a contradiction; if $g$ is odd, $p \geq \frac{g+1}{2}$ and $q \leq \frac{g-1}{2}$, also a contradiction. Hence, $q=\left\lceil\frac{g}{2}\right\rceil$ and $p=\left\lfloor\frac{g}{2}\right\rfloor$. Then $G^{*} \cong B\left(G^{*}\right) \cong P\left(\left\lfloor\frac{g}{2}\right\rfloor,\left\lceil\frac{g}{2}\right\rceil,\left\lceil\frac{g}{2}\right\rceil\right)$.

Case 2. $n=g+\left\lceil\frac{g}{2}\right\rceil$. Then $p+q+r \leq g+\left\lceil\frac{g}{2}\right\rceil+1$. By (3.1), we have $\left\lceil\frac{g}{2}\right\rceil \leq r \leq\left\lceil\frac{g}{2}\right\rceil+1$ and $p \geq\left\lfloor\frac{g}{2}\right\rfloor-1, q \leq\left\lceil\frac{g}{2}\right\rceil+1$.

If $r=\left\lceil\frac{g}{2}\right\rceil$, similar to Case 1 , we have $q=\left\lceil\frac{g}{2}\right\rceil$ and $p=\left\lfloor\frac{g}{2}\right\rfloor$.

If $r=\left\lceil\frac{g}{2}\right\rceil+1$, then $G^{*}$ cannot contain pendant edges. If $g=3,\left\{\begin{array}{l}p=\left\lfloor\frac{g}{2}\right\rfloor=1 \\ q=\left\lceil\frac{g}{2}\right\rceil=2\end{array}\right.$ since $p \geq 1$; If $g \geq 4,\left\{\begin{array}{l}p=\left\lfloor\frac{g}{2}\right\rfloor-1 \\ q=\left\lceil\frac{g}{2}\right\rceil+1\end{array}\right.$ or $\left\{\begin{array}{l}p=\left\lfloor\frac{g}{2}\right\rfloor \\ q=\left\lceil\frac{g}{2}\right\rceil\end{array}\right.$.

Case 2.1. $g=3$. Then $G^{*}$ is obtained from $P(1,2,2)$ by attaching a pendant edge to a vertex of $P(1,2,2)$ or $G^{*} \cong P(1,2,3)$. By direct calculation, we have

$$
\mu\left(P(1,2,2) v S_{2}\right)=5.7785, \quad \mu(P(1,2,3))=5.1149 .
$$

Then $B\left(G^{*}\right) \cong P(1,2,2)$.

Case 2.2. $g=4$. Then $G^{*}$ is obtained from $P(2,2,2)$ by attaching a pendant edge to a vertex of $P(2,2,2)$ or $G^{*} \in\{P(1,3,3), P(2,2,3)\}$. By direct calculation, we have

$$
\mu\left(P(2,2,2) v S_{2}\right)=5.5141, \quad \mu(P(1,3,3))=5.0000, \quad \mu(P(2,2,3))=4.9032 .
$$


Then $B\left(G^{*}\right) \cong P(2,2,2)$.

Case 2.3. $g=5$. Then $G^{*}$ is obtained from $P(2,3,3)$ by attaching a pendant edge to a vertex of $P(2,3,3)$ or $G^{*} \in\{P(1,4,4), P(2,3,4)\}$. By direct calculation, we have

$$
\mu\left(P(2,3,3) v S_{2}\right)=5.3552, \quad \mu(P(1,4,4))=4.9032, \quad \mu(P(2,3,4))=4.7728 .
$$

Then $B\left(G^{*}\right) \cong P(2,3,3)$.

Case 2.4. $g \geq 6$. Then $G$ is obtained from $P\left(\left\lfloor\frac{g}{2}\right\rfloor,\left\lceil\frac{g}{2}\right\rceil,\left\lceil\frac{g}{2}\right\rceil\right)$ by attaching a pendant edge to a vertex of $P\left(\left\lfloor\frac{g}{2}\right\rfloor,\left\lceil\frac{g}{2}\right\rceil,\left\lceil\frac{g}{2}\right\rceil\right)$ or $G \in\left\{P\left(\left\lfloor\frac{g}{2}\right\rfloor-1,\left\lceil\frac{g}{2}\right\rceil+1,\left\lceil\frac{g}{2}\right\rceil+\right.\right.$ 1), $\left.P\left(\left\lfloor\frac{g}{2}\right\rfloor,\left\lceil\frac{g}{2}\right\rceil,\left\lceil\frac{g}{2}\right\rceil+1\right)\right\}$. By Lemma 2.6, we have

$$
\begin{aligned}
\mu\left(P\left(\left\lfloor\frac{g}{2}\right\rfloor-1,\left\lceil\frac{g}{2}\right\rceil+1,\left\lceil\frac{g}{2}\right\rceil+1\right)\right) & \leq 5, \\
\mu\left(P\left(\left\lfloor\frac{g}{2}\right\rfloor,\left\lceil\frac{g}{2}\right\rceil,\left\lceil\frac{g}{2}\right\rceil+1\right)\right) & \leq 5 .
\end{aligned}
$$

And by Lemma 2.5, we have

$$
\mu\left(P\left(\left\lfloor\frac{g}{2}\right\rfloor,\left\lceil\frac{g}{2}\right\rceil,\left\lceil\frac{g}{2}\right\rceil\right) v S_{2}\right)>5 .
$$

Then $B\left(G^{*}\right) \cong P\left(\left\lfloor\frac{g}{2}\right\rfloor,\left\lceil\frac{g}{2}\right\rceil,\left\lceil\frac{g}{2}\right\rceil\right)$.

Case 3. $n \geq g+\left\lceil\frac{g}{2}\right\rceil+1$.

If $r=\left\lceil\frac{g}{2}\right\rceil$, Similar to Case 1, we have $q=\left\lceil\frac{g}{2}\right\rceil$ and $p=\left\lfloor\frac{g}{2}\right\rfloor$.

If $r \geq\left\lceil\frac{g}{2}\right\rceil+1$, let $k=n-(p+q+r)+1$, that is, the number of pendant edges in $G^{*}$. Then $k \leq n-g-\left\lceil\frac{g}{2}\right\rceil$. It is easy to see that $\max \left\{d_{x}+m_{x} \mid x \in V\left(G^{*}\right)\right\}$ attains the maximum just when $p=1$ and $k$ pendent edges of $G^{*}$ are incident to a 3 -degree vertex of $P(p, q, r)$. In this case, by Lemma 2.6 , we have

$$
\begin{aligned}
\mu(G) \leq \max \left\{d_{x}+m_{x} \mid x \in V(G)\right\} & =k+3+\frac{k+7}{k+3} \\
& <n-g-\left\lceil\frac{g}{2}\right\rceil+4+\frac{4}{n-g-\left\lceil\frac{g}{2}\right\rceil+3} \\
& \leq n-g-\left\lceil\frac{g}{2}\right\rceil+5,
\end{aligned}
$$

since $k+3+\frac{k+7}{k+3}$ is increasing with nonnegative number $k$. However, by Lemma 2.5 ,

$$
\mu\left(P\left(\left\lfloor\frac{g}{2}\right\rfloor,\left\lceil\frac{g}{2}\right\rceil,\left\lceil\frac{g}{2}\right\rceil\right) v S_{n-g-\left\lceil\frac{g}{2}\right\rceil+2}\right)>n-g-\left\lceil\frac{g}{2}\right\rceil+5 .
$$

Then $B\left(G^{*}\right) \cong P\left(\left\lfloor\frac{g}{2}\right\rfloor,\left\lceil\frac{g}{2}\right\rceil,\left\lceil\frac{g}{2}\right\rceil\right)$.

TheOREM 3.6. Let $G^{*}$ have the maximal signless Laplacian spectral radius among all graphs in $B_{2}(n, g), n \geq g+\left\lceil\frac{g}{2}\right\rceil-1$. Then $G^{*} \cong P\left(\left\lfloor\frac{g}{2}\right\rfloor,\left\lceil\frac{g}{2}\right\rceil,\left\lceil\frac{g}{2}\right\rceil\right) v S_{n-g-\left\lceil\frac{g}{2}\right\rceil+2}$. 
Proof. Let $k=n-g-\left\lceil\frac{g}{2}\right\rceil+1$. By Lemma 3.3 and Lemma 3.5, we know that $G^{*}$ is obtained from $P\left(\left\lfloor\frac{g}{2}\right\rfloor,\left\lceil\frac{g}{2}\right\rceil,\left\lceil\frac{g}{2}\right\rceil\right)$ by attaching $k$ pendant edges to a unique vertex $w$.

Case 1. $k=0$. Then $G^{*} \cong P\left(\left\lfloor\frac{g}{2}\right\rfloor,\left\lceil\frac{g}{2}\right\rceil,\left\lceil\frac{g}{2}\right\rceil\right)$.

Case 2. $k=1$.

Case 2.1. $g=3$. Let $A_{1}$ be the graph obtained from $P(1,2,2)$ by attaching a pendant edge to a 2 -degree vertex of $P(1,2,2)$. Since $B_{2}(5,3)=\left\{A_{1}, P(1,2,2) v S_{2}\right.$, $P(1,2,3)\}$, by Lemma 3.5 , we have

$$
G^{*} \in\left\{A_{1}, P(1,2,2) v S_{2}\right\}
$$

By direct calculation, we have

$$
\mu\left(A_{1}\right)=5.4679, \quad \mu\left(P(1,2,2) v S_{2}\right)=5.7785 .
$$

Case 2.2. $g=4$. Let $A_{2}$ be the graph obtained from $P(2,2,2)$ by attaching a pendant edge to a 2-degree vertex of $P(2,2,2)$. Since $B_{2}(6,4)=\left\{A_{2}, P(2,2,2) v S_{2}\right.$, $P(1,3,3), P(2,2,3)\}$, by Lemma 3.5 , we have

$$
G^{*} \in\left\{A_{2}, P(2,2,2) v S_{2}\right\}
$$

By direct calculation, we have

$$
\mu\left(A_{2}\right)=5.2361, \quad \mu\left(P(2,2,2) v S_{2}\right)=5.5141
$$

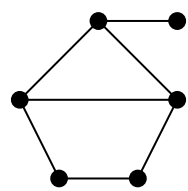

$A_{3}$

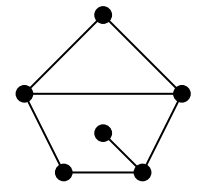

$A_{4}$

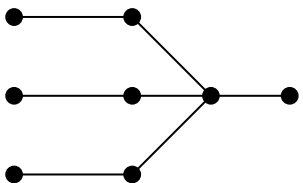

$A_{5}$

FIG. 3.3. The graphs $A_{3}, A_{4}$ and $A_{5}$.

Case 2.3. $g=5$. Let $A_{3}, A_{4}$ be the graphs as shown in Figure 3.3. Since

$$
B_{2}(8,5)=\left\{A_{3}, A_{4}, P(2,3,3) v S_{2}, P(1,4,4), P(2,3,4)\right\},
$$

by Lemma 3.5, we have

$$
G^{*} \in\left\{A_{3}, A_{4}, P(2,3,3) v S_{2}\right\} .
$$


By direct calculation, we have

$$
\mu\left(A_{3}\right)=5.0664, \quad \mu\left(A_{4}\right)=4.9891, \quad \mu\left(P(2,3,3) v S_{2}\right)=5.3552 .
$$

Case 2.4. $g \geq 6$. By Lemma 3.5, we have $B\left(G^{*}\right) \cong P\left(\left\lfloor\frac{g}{2}\right\rfloor,\left\lceil\frac{g}{2}\right\rceil,\left\lceil\frac{g}{2}\right\rceil\right)$. It is easy to see that $w$ cannot be simultaneously adjacent to the two 3 -degree vertices of $P\left(\left\lfloor\frac{g}{2}\right\rfloor,\left\lceil\frac{g}{2}\right\rceil,\left\lceil\frac{g}{2}\right\rceil\right)$ and these two 3-degree vertices are not adjacent. If $G \neq$ $P\left(\left\lfloor\frac{g}{2}\right\rfloor,\left\lceil\frac{g}{2}\right\rceil,\left\lceil\frac{g}{2}\right\rceil\right) v S_{2}$, by Lemma 2.7 , we have

$$
\begin{aligned}
\mu(G) & \leq \max \left\{\frac{d_{v_{i}}\left(d_{v_{i}}+m_{v_{i}}\right)+d_{v_{j}}\left(d_{v_{j}}+m_{v_{j}}\right)}{d_{v_{i}}+d_{v_{j}}} \mid v_{i} v_{j} \in E(G)\right\} \\
& \leq \max \left\{\frac{31}{6}, \frac{26}{5}, \frac{20}{4}\right\}=\frac{26}{5} .
\end{aligned}
$$

But

$$
\mu\left(P\left(\left\lfloor\frac{g}{2}\right\rfloor,\left\lceil\frac{g}{2}\right\rceil,\left\lceil\frac{g}{2}\right\rceil\right) v S_{2}\right) \geq \mu\left(A_{5}\right)=5.2361 .
$$

Then $G^{*} \cong P\left(\left\lfloor\frac{g}{2}\right\rfloor,\left\lceil\frac{g}{2}\right\rceil,\left\lceil\frac{g}{2}\right\rceil\right) v S_{2}$.

Case 3. $k \geq 2$. By Lemma 3.5, we have $B\left(G^{*}\right) \cong P\left(\left\lfloor\frac{g}{2}\right\rfloor,\left\lceil\frac{g}{2}\right\rceil,\left\lceil\frac{g}{2}\right\rceil\right)$. If $G^{*} \neq$ $P\left(\left\lfloor\frac{g}{2}\right\rfloor,\left\lceil\frac{g}{2}\right\rceil,\left\lceil\frac{g}{2}\right\rceil\right) v S_{k+1}$, by Lemma 2.6, we have

$$
\mu\left(G^{*}\right) \leq k+2+\frac{k+6}{k+2} \leq k+4
$$

since $k \geq 2$. But by Lemma 2.5 ,

$$
\mu\left(P\left(\left\lfloor\frac{g}{2}\right\rfloor,\left\lceil\frac{g}{2}\right\rceil,\left\lceil\frac{g}{2}\right\rceil\right) v S_{k+1}\right)>k+4,
$$

since $P\left(\left\lfloor\frac{g}{2}\right\rfloor,\left\lceil\frac{g}{2}\right\rceil,\left\lceil\frac{g}{2}\right\rceil\right) v S_{k+1}$ is not a star. Then $G^{*} \cong P\left(\left\lfloor\frac{g}{2}\right\rfloor,\left\lceil\frac{g}{2}\right\rceil,\left\lceil\frac{g}{2}\right\rceil\right) v S_{k+1}$. $\square$

THEOREM 3.7. Let $G^{*}$ have the maximal signless Laplacian spectral radius among all graphs in $B(n, g), n \geq g+\left\lceil\frac{g}{2}\right\rceil-1$. Then $G^{*} \cong P\left(\left\lfloor\frac{g}{2}\right\rfloor,\left\lceil\frac{g}{2}\right\rceil,\left\lceil\frac{g}{2}\right\rceil\right) v S_{n-g-\left\lceil\frac{g}{2}\right\rceil+2}$ and $\mu\left(G^{*}\right)<n-g-\left\lceil\frac{g}{2}\right\rceil+5+\frac{4}{n-g-\left\lceil\frac{g}{2}\right\rceil+4}$.

Proof. Case 1. $g=3$. By Lemma 2.8, we have

$$
\mu\left(\widehat{B}(3,1,3) v_{1} S_{n-4}\right)<\mu\left(P(1,2,2) v S_{n-3}\right) .
$$

Case 2. $g \geq 4$. By Theorem 3.4,

$$
\mu\left(\widehat{B}(g, 1, g) v_{1} S_{n-2 g+2}\right)<n-2 g+6+\frac{4}{n-2 g+5} \leq n-2 g+7,
$$

since in this case, $n \geq 2 g-1$. But by Lemma 2.5 ,

$$
\mu\left(P\left(\left\lfloor\frac{g}{2}\right\rfloor,\left\lceil\frac{g}{2}\right\rceil,\left\lceil\frac{g}{2}\right\rceil\right) v S_{n-g-\left\lceil\frac{g}{2}\right\rceil+2}\right)>\Delta+1=n-g-\left\lceil\frac{g}{2}\right\rceil+5,
$$


since $P\left(\left\lfloor\frac{g}{2}\right\rfloor,\left\lceil\frac{g}{2}\right\rceil,\left\lceil\frac{g}{2}\right\rceil\right) v S_{n-g-\left\lceil\frac{g}{2}\right\rceil+2}$ is not a star. However

$$
n-g-\left\lceil\frac{g}{2}\right\rceil+5-(n-2 g+7)=\left\lfloor\frac{g}{2}\right\rfloor-2 \geq 0,
$$

since $g \geq 4$. Hence,

$$
\mu\left(\widehat{B}(g, 1, g) v_{1} S_{n-2 g+2}\right)<\mu\left(P\left(\left\lfloor\frac{g}{2}\right\rfloor,\left\lceil\frac{g}{2}\right\rceil,\left\lceil\frac{g}{2}\right\rceil\right) v S_{n-g-\left\lceil\frac{g}{2}\right\rceil+2}\right) .
$$

So $G^{*} \cong P\left(\left\lfloor\frac{g}{2}\right\rfloor,\left\lceil\frac{g}{2}\right\rceil,\left\lceil\frac{g}{2}\right\rceil\right) v S_{n-g-\left\lceil\frac{g}{2}\right\rceil+2}$. Furthermore, by Lemma 2.6, we have

$$
\mu\left(G^{*}\right)<n-g-\left\lceil\frac{g}{2}\right\rceil+5+\frac{4}{n-g-\left\lceil\frac{g}{2}\right\rceil+4} .
$$

Acknowledgment. The author is grateful to the referee for his/her valuable comments, corrections and suggestions, which led to an improved version of the original manuscript.

\section{REFERENCES}

[1] B. Bollobás. Modern Graph Theory. Springer-Verlag, New York, 1998.

[2] E.R. van Dam and W. Haemers. Which graphs are determined by their spectrum? Linear Algebra Appl., 373:241-272, 2003.

[3] X.D. Zhang. The signless Laplacian spectral radius of trees with given degree sequences. Discrete Math., 308:3143-3150, 2008.

[4] X.D. Zhang. The signless Laplacian spectral radius of graphs with given degree sequences. Discrete Appl. Math., 157:2928-2937, 2009.

[5] Y.Z. Fan, B.S. Tam, and J. Zhou. Maximizing spectral radius of unoriented Laplacian matrix over bicyclic graphs. Linear and Multilinear Algebra, 56:381-397, 2008.

[6] L. Feng, Q. Li, and X. Zhang. Some sharp upper bounds on the spectral radius of graphs. Taiwanese J. Math., 11:989-997, 2007.

[7] D. Cvetković. Spectral theory of graphs based on the signless Laplacian. Research report. Availiable at www.mi.sanu.ac.rs/projects/signless_L_reportJan28.pdf.

[8] A.J. Hoffman and J.H. Smith. On the spectral radii of topologically equivalent graphs. In M. Fiedler (editor), Recent Advances in Graph Theory, Academia Praha, New York, 273-281, 1975.

[9] D. Cvetković and S.K. Simic. Towards a spectral theory of graphs based on the signless Laplacian. Publ. Inst. Math. (Beograde), 85(99):19-33, 2009.

[10] L. Feng, Q. Li, and X. Zhang. Minimizing the Laplacian spectral radius of trees with given matching number. Linear and Multilinear Algebra, 55:199-207, 2007.

[11] L. Feng. The signless Laplacian spectral radius for bicyclic graphs with $k$ pendant vertices. Kyungpook Math. J., 50:109-116, 2010.

[12] D. Cvetković, P. Rowlinson, and S.K. Simic. Eigenvalue bounds for the signless Laplacian. Publ. Inst. Math. (Beograde), 81(99):11-27, 2007.

[13] C.S. Oliveira, L.S. de Lima, N.M.M. de Abreu, and P. Hansen. Bounds on the index of the signless Laplacian of a graph. Discrete Appl. Math., 158:355-360, 2010. 